\title{
Oxidación Selectiva de Sulfuros a Sulfóxidos y Sulfonas utilizando un Nuevo Catalizador con Estructura tipo Keggin $\left(\mathrm{H}_{5} \mathrm{PMO}_{11} \mathrm{Al}_{0.5} \mathrm{~V}_{0.5} \mathrm{O}_{40}\right)$
}

Gustavo Romanelli (1)*, Patricia Vázquez (1), Pablo Dimitroff (1) y Pietro Tundo (2)

1) Centro de Investigación y Desarrollo en Ciencias Aplicadas "Dr. Jorge J. Ronco" (CINDECA) CONICET-Universidad de La Plata, Calle $47 \mathrm{~N}^{\text {ro }} 257$ (B1900AJK) La Plata-Argentina (e-mail: gpr@quimica.unlp.edu.ar)

2) Consorzio Interuniversitario Nazionale La Chimica per l'Ambiente, Dorsoduro 2137-30123 Venezia, Italia

* autor a quien debe ser dirigida la correspondencia

\section{Resumen}

En esta investigación, se ha encontrado un nuevo y selectivo procedimiento de oxidación de sulfuros aromáticos y alifáticos, en sulfóxidos y sulfonas, empleando $35 \% \mathrm{H}_{2} \mathrm{O}_{2}$ y acetonitrilo como solvente de reacción. Esta oxidación selectiva se realiza en presencia de cantidades catalíticas de un nuevo heteropoliácido, $\mathrm{H}_{5} \mathrm{PMO}_{11} \mathrm{Al}_{0.5} \mathrm{~V}_{0.5} \mathrm{O}_{40}$, con estructura tipo Keggin. Los rendimientos fueron excelentes y todos los reactivos son baratos y de fácil acceso. La reacción para la preparación de sulfóxidos fue llevada a cabo a temperatura ambiente y a cortos tiempos. La oxidación a sulfotas procede a $40 \mathrm{C}$ dando también excelentes rendimientos.

Palabras clave: heteropoliácidos tipo Keggin, peróxido de hidrógeno, oxidación, sulfóxidos, sulfonas

\section{Selective Oxidation of Sulfides to Sulfoxides and Sulfones using a New Catalyst With Keggin type-Structure $\left(\mathrm{H}_{5} \mathrm{PMo}_{11} \mathrm{Al}_{0.5} \mathrm{~V}_{0.5} \mathrm{O}_{40}\right)$}

\begin{abstract}
In this research, a new and selective procedure for the oxidation of sulfides, aromatics and aliphatics, to sulfoxides and sulfones, with $35 \%$ aqueous $\mathrm{H}_{2} \mathrm{O}_{2}$ and acetonitrile as solvent. Catalytic amount of new Keggin heteropolyacid $\mathrm{H}_{5} \mathrm{PMo}_{11} \mathrm{Al}_{0.5} \mathrm{~V}_{0.5} \mathrm{O}_{40}$ were used. The yields were excellent and all reagents are cheap and easily available. The reaction for preparation of sulfoxides was carried out at room temperature and short times. The oxidation to sulfones proceeded at $40{ }^{\circ} \mathrm{C}$ in also excellent yields.
\end{abstract}

Keywords: Keggin heteropolyacid, hydrogen peroxide, oxidation, sulfoxides, sulfones 


\section{INTRODUCCIÓN}

La conversión selectiva de sulfuros en sulfóxidos es una transformación de gran importancia en la industria y en investigación básica. Ha sido estudiada desde mediados del siglo XIX debido a la importancia de los sulfóxidos como intermediarios en síntesis orgánica. En el año 1865, Maercker realizó la primera síntesis de sulfóxidos y a partir de esa fecha numerosos métodos han sido desarrollados para esta reacción (Kar et al., 2003).

Como describe Bonadis, agentes oxidantes como el ozono, perácidos, ácido crómico, nítrico, dióxido de manganeso, periodato de sodio, dióxido de selenio, perborato de sodio, t-butilhidroperóxido, entre otros, han sido utilizados con este propósito (Bonadies et al., 1996). En los últimos años se han desarrollado diferentes sistemas catalíticos, entre ellos los heteropoliácidos (HPA) (Xu et al., 2004).

Por otra parte, las sulfonas son importantes intermediarios en la síntesis de moléculas orgánicas más complejas. Existen diferentes métodos para su preparación a partir de sulfuros, la mayoría de ellos emplean reactivos similares a los utilizados en la oxidación de sulfuros a sulfóxidos (Alonso et al., 2003).

En la mayoría de los métodos descriptos en literatura y resumidos por Bonadies se generan contaminantes, ya que los reactivos involucrados son ácidos corrosivos, sustancias tóxicas o peligrosas. (Bonadies et al., 1996). Por tal motivo se presenta la necesidad de implementar tecnologías limpias y el peróxido de hidrógeno se distingue como atractivo reactivo para la oxidación de sustratos orgánicos debido a la eficiencia en su contenido de oxigeno y su bajo costo. Debe destacarse que en las reacciones con peróxido de hidrógeno el único subproducto es el agua.

La gran demanda de procesos compatibles con el medio ambiente ha llevado a un esfuerzo de la comunidad científica para utilizar materiales no contaminantes y los HPA han atraído considerable atención por su baja toxicidad y sus manifiestas propiedades redox, incluyendo su acidez. Recientemente, nuestro grupo ha estudiado reacciones de oxidación con peróxido de hidrógeno acuoso de fenoles (Villabrille et al., 2004) y sulfuros con otros catalizadores de estructura tipo Keggin (Romanelli et al., 2005).

En la presente investigación se estudia la influencia de diferentes HPAs tipo Keggin que contienen en su estructura fósforo $y$ molibdeno, sobre las reacciones de oxidación catalítica, con peróxido de hidrógeno acuoso, de sulfuros a sulfóxidos y a sulfonas, de acuerdo a lo presentado en el Esquema 1 (Figura 1). Particularmente, se presenta el $\mathrm{H}_{5} \mathrm{PMo}_{11} \mathrm{Al}_{0.5} \mathrm{~V}_{0.5} \mathrm{O}_{40}$ (HPAA) como nuevo heteropolicatalizador para este tipo de reacciones.

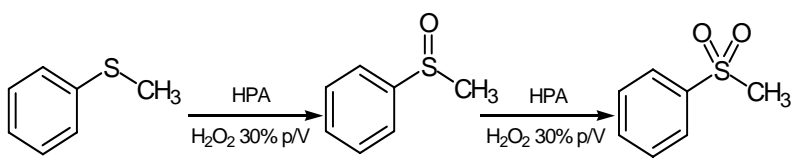

Fig. 1: Esquema de oxidación catalítica

\section{EXPERIMENTAL}

Síntesis y caracterización del HPAA.

Una mezcla estequiométrica de $0,58 \mathrm{~g}$ de $\mathrm{H}_{3} \mathrm{PO}_{4} \quad 85 \%$ (Mallinckrodt), 0,603 $\mathrm{g}$ de $\mathrm{AlCl}_{3} \cdot 6 \mathrm{H}_{2} \mathrm{O}, 0,455 \mathrm{~g}$ de $\mathrm{V}_{2} \mathrm{O}_{5}$ y $14,4 \mathrm{~g}$ de $\mathrm{MoO}_{3}$ (Mallinckrodt) se adicionaron en $150 \mathrm{ml}$ de agua destilada. La mezcla se agitó durante $3 \mathrm{~h}$, a $80^{\circ} \mathrm{C}$, obteniéndose cristales de color naranja. Los diagramas de rayos $X$ (DRX) se realizaron con un equipo Philips modelo PW1714 con registrador gráfico de barrido. Se usó radiación $\mathrm{Cu} \mathrm{K}_{\alpha}, 30 \mathrm{~mA}$ y $40 \mathrm{kV}$ en la fuente de alta tensión. La determinación de acidez mediante Titulación Potenciométrica se llevó a cabo mediante el agregado de 0,05 $\mathrm{ml}$ de una solución de $\mathrm{n}$-butilamina $(0,05 \mathrm{~N})$ a $0,05 \mathrm{~g}$ del sólido de interés previamente suspendido en acetonitrilo. El potencial $(\mathrm{mV})$ fue medido en un pHmetro digital Instrumentalia S.R.L, con un electrodo combinado de $\mathrm{Ag} / \mathrm{AgCl}$, con punta de platino. Para Infrarrojo con Transformada de Fourier (FT-IR) se usó un equipo Bruker IFS 66, midiendo en el rango de $4000-400 \mathrm{~cm}^{-1}$, para obtener los espectros de distintas muestras preparadas en forma de pastillas en $\mathrm{KBr}$.

Oxidación catalítica. Proceso general para la preparación de sulfóxidos. Se agita una solución del sulfuro correspondiente $(0,7$ mmoles) y el catalizador HPAA $(0,01$ mmoles), en $5 \mathrm{ml}$ de acetonitrilo. Se agrega 1 
$\mathrm{ml}$ de $\mathrm{H}_{2} \mathrm{O}_{2}\left(30 \%(\mathrm{p} / \mathrm{v})\right.$, a $20{ }^{\circ} \mathrm{C}$. La solución se agita por un determinado período de tiempo, dependiendo del tipo de sustrato. Posteriormente, se evapora el solvente y el residuo se extrae con $10 \mathrm{ml}$ de $\mathrm{CH}_{2} \mathrm{Cl}_{2}$, el extracto orgánico se seca con $\mathrm{Na}_{2} \mathrm{SO}_{4}$ anhidro, se filtra el desecante y se evapora el solvente. Los sulfóxidos sólidos se purificaron por recristalización. Todos los productos fueron identificados por espectrometría de masas.

Proceso general para la preparación de sulfonas. Se agita una solución del sulfuro correspondiente $(0,7$ mmoles $)$ y HPAA $(0,01$ mmoles), en $5 \mathrm{ml}$ de acetonitrilo. Se agrega 1 $\mathrm{ml}$ de $\mathrm{H}_{2} \mathrm{O}_{2}(30 \%(\mathrm{p} / \mathrm{v}))$, a $40^{\circ} \mathrm{C}$. La solución se agita por un período de tiempo entre 2 y 4 $\mathrm{h}$, dependiendo del tipo de sustrato. Se evapora el solvente. El residuo se extrae con $10 \mathrm{ml}$ de $\mathrm{CH}_{2} \mathrm{Cl}_{2}$, el extracto orgánico se seca con $\mathrm{Na}_{2} \mathrm{SO}_{4}$ anhidro, se filtra el desecante y se evapora el solvente. Los sólidos se purificaron por recristalización. Como en el ítem anterior los productos fueron identificados por espectrometría de masas. El rendimiento indicado el las tablas es del producto aislado y puro.

\section{RESULTADOS Y DISCUSIÓN}

Las propiedades de oxidación de los HPA tipo Keggin pueden modificarse por la adecuada elección del heteroátomo, de los oxometales de la estructura primaria y del catión. La síntesis de compuestos tipo $\left[\mathrm{PMo}_{12-n} \mathrm{Al}_{\mathrm{n}} \mathrm{V}_{\mathrm{n}} \mathrm{O}_{40}\right]^{-\mathrm{x}}$, donde $\mathrm{n}$ puede tener valores de 1 a 12 , está orientada a la búsqueda de propiedades redox que aumenten su performance catalítica.

El espectro FT-IR del $\mathrm{H}_{3} \mathrm{PMo}_{12} \mathrm{O}_{40}$ presenta señales características debido a sus enlaces:

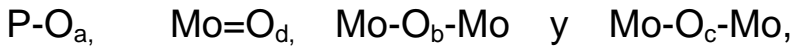
respectivamente. Estas señales se dan a: $1064 \mathrm{~cm}^{-1}\left(\mathrm{P}-\mathrm{O}_{\mathrm{a}}\right) ; 964 \mathrm{~cm}^{-1}\left(\mathrm{Mo}=\mathrm{O}_{\mathrm{d}}\right) ; 871 \mathrm{~cm}^{-1}$ $\left(\mathrm{Mo}-\mathrm{O}_{\mathrm{b}}-\mathrm{Mo}\right) ; 784 \mathrm{~cm}^{-1} \quad\left(\mathrm{Mo}_{\mathrm{C}}-\mathrm{O} \mathrm{O}\right)$. El espectro vibracional de los ácidos másicos, con estructura Keggin, se ve modificado de acuerdo a la naturaleza de los elementos que se introducen en estos compuestos. Las modificaciones más significativas cuando un metal es introducido en la estructura compuesta por Mo son una disminución en la frecuencia del modo $\mathrm{M}=\mathrm{O}_{\mathrm{d}}\left(964 \mathrm{~cm}^{-1}\right)$ y un posible desdoblamiento para la banda del $\mathrm{P}$ $\mathrm{O}_{\mathrm{a}}\left(1064 \mathrm{~cm}^{-1}\right)$. La presencia de un átomo de
Al en la estructura del $\mathrm{H}_{3} \mathrm{PMo}_{12} \mathrm{O}_{40}$ no implica modificaciones de tipo estructural, como se ha observado mediante estudios de FT-IR y DRS, pero la diferencia de cargas originada por la presencia de otros $\mathrm{H}$ involucra un reacomodamiento de las posiciones de las aguas de hidratación en la estructura primaria de este ácido (Villabrille et al., 2004). En los diagramas de DRX para el HPAA, a temperatura ambiente y hasta los $180{ }^{\circ} \mathrm{C}$ el diagrama de la estructura de Keggin se encuentra intacta. A partir de esta temperatura, comienza a cambiar hacia estructuras modificadas de molibdeno, aluminio y vanadio, aunque no se pierden las propiedades ácidas del compuesto original, lo que fue determinado por titulación potenciométrica.

Inicialmente se optimizaron las condiciones de reacción utilizando fenil metil sulfuro como sustrato (Figura 1). Las conversiones y la selectividad de las diferentes reacciones fueron estimadas por cromatografía gaseosaespectrometría de masas. En los diferentes análisis llevados a cabo solo se detecta sulfuro, sulfóxido y sulfona, no observándose formación de otros productos secundarios.

La reacción produce conversiones muy bajas sin catalizador ( $10 \%$ de sulfóxido para $20 \mathrm{~h}$, a $20^{\circ} \mathrm{C}$ ). Al emplear HPA comerciales, $\mathrm{H}_{3} \mathrm{PMo}_{12} \mathrm{O}_{40}$ y $\mathrm{H}_{4} \mathrm{SiMo}_{12} \mathrm{O}_{40}$, se observa un incremento en la conversión (95 y $92 \%$, respectivamente, para $2 \mathrm{~h}$ de reacción, con una selectividad a sulfóxido del $95 \%$, en ambos casos). Posteriormente, se ensayó la actividad catalítica del $\mathrm{H}_{6} \mathrm{PMo}_{11} \mathrm{AlO}_{40}$, un HPA preparado en nuestro laboratorio (resultados aun no publicados). En condiciones de reacción similares a las empleadas con los catalizadores comerciales mencionados anteriormente se alcanza una conversión del 74\% y una selectividad de sulfóxido de 99\%. Como puede observarse, este HPA es menos activo que los comerciales, sin embargo es más selectivo. Frente a estos resultados se usó como catalizador el HPAA. Al incorporar vanadio en la estructura del $\mathrm{H}_{6} \mathrm{PMo}_{11} \mathrm{AlO}_{40}$ se observa un notable incremento en la actividad (conversión del sulfuro del $93 \%$ en solo 20 minutos de reacción, con una selectividad a sulfóxido del $100 \%$ ).

Para optimizar las condiciones de reacción, se estudió el efecto de la temperatura sobre la velocidad de conversión y la selectividad, 
manteniendo constante la relación de $\mathrm{H}_{2} \mathrm{O}_{2}$ /sustrato. A $20{ }^{\circ} \mathrm{C}$ la reacción es selectiva al sulfóxido (99\%) con una conversión del $100 \%$. Al elevar la temperatura, se incrementa la velocidad de conversión y se observa un aumento en la selectividad pero a la sulfona. Por ej., a una temperatura de $40{ }^{\circ} \mathrm{C}$ y $3 \mathrm{~h}$ de reacción, se obtiene una selectividad a sulfona del $100 \%$.

Optimizadas las condiciones de reacción para la oxidación selectiva de sulfuros a sulfóxidos y a sulfonas, se extendió la reacción a otros sustratos. En la Tabla 1 pueden observarse los resultados obtenidos para la oxidación selectiva de seis sulfuros de diferente naturaleza estructural a sulfóxidos y en la Tabla 2 se presentan los resultados obtenidos para la oxidación selectiva de los mismos seis sulfuros en este caso a sulfonas. Cuando la reacción se lleva a cabo a $40{ }^{\circ} \mathrm{C}$ al igual que en el caso de los sulfóxidos los rendimientos de reacción fueron muy buenos.

Tabla 1: Oxidación selectiva de sulfuros a sulfóxidos, en acetonitrilo, a $20{ }^{\circ} \mathrm{C}$

\begin{tabular}{|c|c|c|c|}
\hline Sulfuro & Sulfóxido & $\begin{array}{l}\text { Tiempo } \\
\text { (h) }\end{array}$ & $\begin{array}{l}\text { Rto } \\
\text { (\%) }\end{array}$ \\
\hline Dibutil sulfuro & Dibutilsulfóxido & 0.5 & 96 \\
\hline $\begin{array}{l}\text { Tetrahidro } \\
\text { tiofeno }\end{array}$ & $\begin{array}{c}\text { Tetrahidro } \\
\text { tiofeno-1-oxido }\end{array}$ & 0.5 & 95 \\
\hline $\begin{array}{l}\text { Fenil metil } \\
\text { sulfuro }\end{array}$ & $\begin{array}{l}\text { Fenil metil } \\
\text { Sulfóxido }\end{array}$ & 1 & 94 \\
\hline Difenil sulfuro & Difenil sulfoxido & 6 & 100 \\
\hline Dibencil sulfuro & Dibencil sulfóxido & 0.5 & 95 \\
\hline $\begin{array}{l}\text { Metil p-tolil } \\
\text { sulfuro }\end{array}$ & $\begin{array}{l}\text { Metil p-tolil } \\
\text { Sulfóxido }\end{array}$ & 1 & 76 \\
\hline
\end{tabular}

Tabla 2: Oxidación selectiva de sulfuros a sulfonas, en acetonitrilo, a $40^{\circ} \mathrm{C}$

\begin{tabular}{cccc}
\hline Sulfuro & Sulfóxido & $\begin{array}{c}\text { Tiempo } \\
(\mathrm{h})\end{array}$ & $\begin{array}{c}\text { Rto } \\
(\%)\end{array}$ \\
\hline Dibutil sulfuro & Dibutil sulfona & 3 & 92 \\
Tetrahidrotiofeno & Sulfolano & 3 & 91 \\
Fenil metil & $\begin{array}{c}\text { Fenil metil } \\
\text { sulfuro }\end{array}$ & 3 & 98 \\
Difenilsona & & \\
Dibencilsuro & Difenilsulfuro & 4 & 95 \\
Metil p-Tolil & $\begin{array}{c}\text { Dibencilsulfona } \\
\text { Metil p-tolil }\end{array}$ & 4 & 93 \\
sulfuro & sulfona & & 51 \\
\hline
\end{tabular}

\section{CONCLUSIONES}

El catalizador $\mathrm{H}_{5} \mathrm{PMo}_{11} \mathrm{Al}_{0.5} \mathrm{~V}_{0.5} \mathrm{O}_{40}$ fue utilizado para oxidar selectivamente diferentes sulfuros a sulfóxidos y a sulfonas con excelentes rendimientos en condiciones de reacción suaves y sin formación de productos secundarios. La oxidación selectiva de sulfuros a sulfóxidos se llevó a cabo a $20{ }^{\circ} \mathrm{C}$ mientras que la oxidación a sulfona a $40{ }^{\circ} \mathrm{C}$. Además, el método representa una alternativa limpia ya que el agente oxidante utilizado fue peróxido de hidrógeno acuoso.

\section{AGRADECIMIENTOS}

Los autores agradecen a INCA, CONICET; Fundación Antorchas y a la Universidad de La Plata por el apoyo financiero otorgado.

\section{REFERENCIAS}

Alonso, D., C. Nájera y M. Varea, Simple, economical and environmentally friendly sulfone synthesis, Tetrahedron Lett. 43, 34593461 (2003).

Bonadies, F., F. De Angelis, L. Locati y A. Scettri, A convenient acid-catalyzed oxidation of sulfides to sulfoxides by t-butyl hydroperoxide, Tetrahedron Lett. 37, 31293130 (2003).

Kar, G., A. Saikia, U. Bora, S. Dehury y M. Chaudhuri, Synthesis of cetyltrimethylammonium tribromide (CTMATB) and its application in the selective oxidation of sulfides to sulfóxidos, Tetrahedron Lett. 44, 4503-4505 (2003).

Romanelli, G., P. Vázquez, y P. Tundo, New Heteropolyacids as catalysts for the selective oxidation of sulfides to sulfoxides with hydrogen peroxide, Synlett 1, 175-178 (2005).

Romanelli, G., P. Villabrille, C. Cáceres, P. Vázquez y $P$. Tundo, Oxidación de alcoholes, con peróxido de hidrógeno, usando heteropolicompuestos tipo Keggin, 13 Congreso Brasileiro de Catálise, 1130-1135, Foz de Iguacu, Brasil, (2005).

Villabrille, P., G. Romanelli, P. Vázquez y C. Cáceres, Vanadium-substituted Keggin heteropolycompounds as catalysts for ecofriendly liquid phase oxidation of 2,6 Dimethylphenol to 2,6-dimethyl-1,4benzoquinone, Applied Catal. A General 270, 101-110 (2004).

Xu, W., Y. Li, Q. Zhang y H. Zhu, A selective, convenient and efficient conversion of sulfides to sulfoxides, Synthesis 2, 227-232 (2004). 